\title{
Synovial Chondromatosis of the Temporomandibular Joint With Intracranial Extension
}

\author{
-Case Report-
}

\author{
Naoki YOKOTA, Chikanori INENAGA, Tsutomu TOKUYAMA, \\ Shigeru NiSHIZAWA*, Katsutoshi MIURA**, and Hiroki NAMBA \\ Department of Neurosurgery, and **Division of Basic Nursing, Health Science, \\ Pathology and Anatomy, Hamamatsu University School of Medicine, Hamamatsu, Shizuoka; \\ *Department of Neurosurgery, University of Occupational and \\ Environmental Health, Kitakyushu, Fukuoka
}

\begin{abstract}
A 52-year-old man presented with an extremely rare case of synovial chondromatosis in the temporomandibular joint (TMJ) with extension into the middle cranial fossa manifesting as swelling and exacerbation of pain. He had a long history of right TMJ disorders. Computed tomography and magnetic resonance imaging showed a mass in the right TMJ with extension into the intracranial part through the destroyed temporal skull base. The preoperative diagnosis was chondrosarcoma or osteosarcoma. The patient underwent surgery via combined trans-zygomatic temporal skull base and pre-auricular approaches and the mass was totally removed. Histological examination found an enormous number of closely packed loose bodies of various sizes, consisting of hyaline chondrocytes. The histological diagnosis was synovial chondromatosis. This rare lesion is difficult to discriminate from chondrosarcoma, so total removal is essential for correct diagnosis and cure.
\end{abstract}

Key words: synovial chondromatosis, temporomandibular joint, intracranial extension, extradural skull base approach

\section{Introduction}

Synovial chondromatosis is a rare benign proliferative lesion, first reported in $1558,{ }^{3)}$ which mostly affects large articular joints of the body, such as knee, hip, and elbow joints, and is a metaplastic but not neoplastic lesion. ${ }^{7}$ ) Synovial chondromatosis may arise from the synovial membrane or the fibro-cartilaginous disc in large joints. ${ }^{7)}$ Synovial chondromatosis of the temporomandibular joint (TMJ) is rare, and was first described in $1776 .{ }^{4)}$ Recently, a large series of 74 cases of synovial chondromatosis in the TMJ was analyzed. ${ }^{14)}$ The etiology of the disease has not been completely clarified, but may involve secondary reactive metaplasia after trauma or chronic abnormal loading leading to detachment of chondrocytes. Obvious history of the facial trauma is rare.

We treated a patient with synovial chondromatosis in the TMJ and extending into the middle cranial

Received September 6, 2007; Accepted February 6, 2008 fossa via combined trans-zygomatic temporal skull base and pre-auricular approaches.

\section{Case Presentation}

A 52-year-old male patient had been suffering from pain in the right TMJ and swelling in the right side of the face. He was treated with medication under the diagnosis of TMJ disorder in a local hospital. His symptoms were exacerbated, so he was examined by computed tomography (CT) and magnetic resonance (MR) imaging which showed an intracranial mass. The patient was referred to our hospital for further work-up on August 31, 2004.

The patient complained of clicking sound, and difficulty and pain in opening the mouth. His right pre-auricular region was swollen. No facial nerve paresis, disturbance of facial sensation, or hearing disturbance was detected.

CT disclosed a cystic mass in the right TMJ, and multiple fine stippled high-density spots in the cyst (Fig. 1A). The lesion extended into the right 

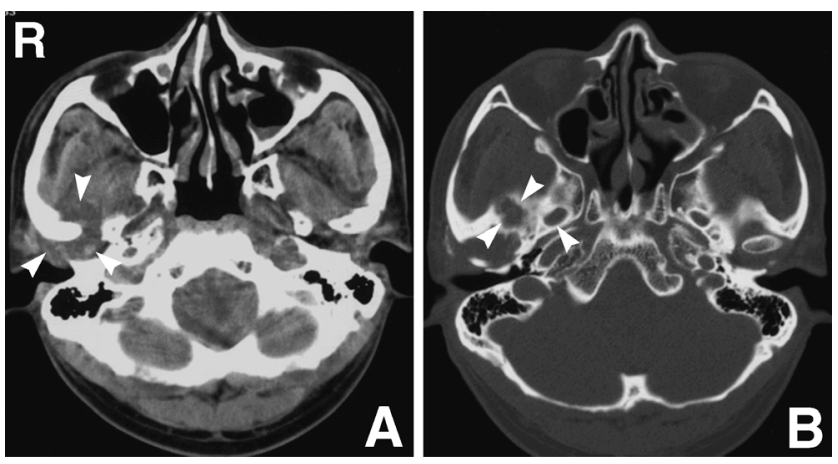

Fig. 1 (A) Computed tomography (CT) scan showing a cystic mass in the right temporomandibular joint (arrowheads) including small calcified nodules. (B) High resolution bone density CT scan demonstrating the destroyed temporal skull base bone (arrowheads), and intact foramina ovale and spinosum.

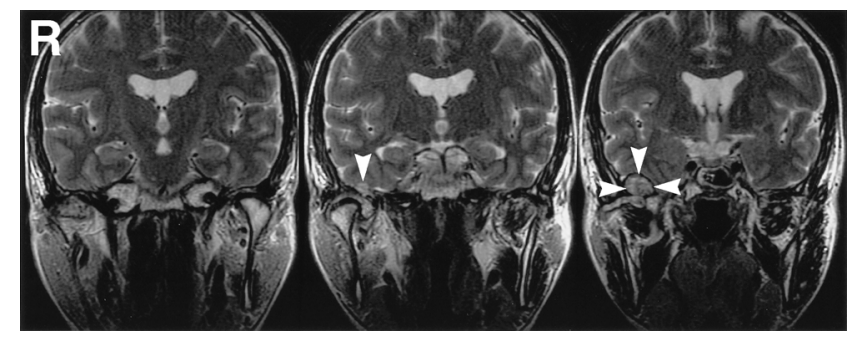

Fig. 2 Coronal $\mathrm{T}_{2}$-weighted magnetic resonance images showing a well-encapsulated cystic mass as mixed density containing both fluid and solid materials (arrowheads), extending into the middle cranial fossa, which continued to the infra-clinoid fossa through the destroyed temporal base bone defect.

middle cranial fossa. Bone density CT revealed destruction of the temporal skull base (Fig. 1B). The foramina ovale and spinosum were intact. Technetium-99m methylene diphosphonate bone scintigraphy showed a well-localized accumulation of the isotope in the right temporomandibular region (not shown). MR imaging also showed a well-encapsulated cystic mass with enhancement of the thick wall by gadolinium-diethylenetriaminepenta-acetic acid. Coronal $\mathrm{T}_{2}$-weighted $\mathrm{MR}$ imaging revealed the mass in the right TMJ and extending into the intracranial part through the destroyed temporal skull base (Fig. 2). The mass appeared as hyperintense, suggesting mucous fluid collection, and contained small hypointense spots. Based on these findings, especially the aggressive bony destruction, a malig-

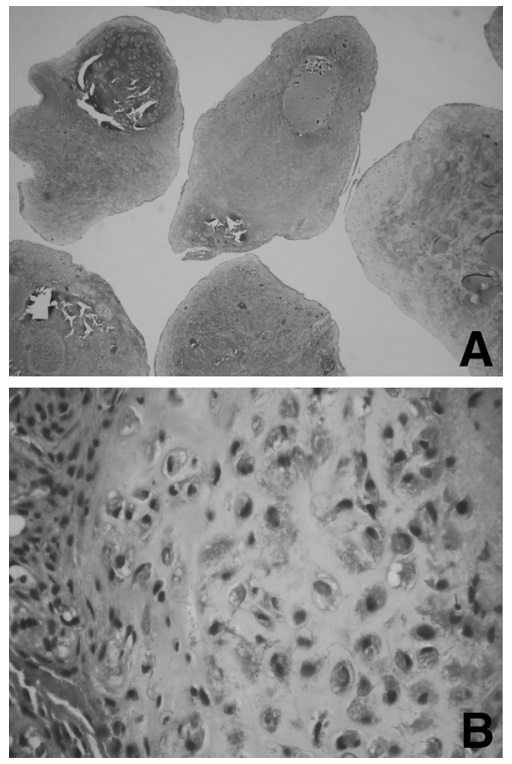

Fig. 3 Photomicrographs of the surgical specimen, showing (A) various sizes of loose bodies, and (B) chondrocytes with some atypia such as nuclear pleomorphism or binucleate cells. A: Hematoxylin and eosin stain, original magnification $\times 40$; $B$ : hematoxylin and eosin stain, original magnification $\times 200$.

nant neoplasm such as chondrosarcoma or osteosarcoma arising from the TMJ was suspected.

The patient underwent surgery via combined right trans-zygomatic temporal skull base and preauricular approaches on September 6, 2004. A frontotemporal skin incision was extended below the zygomatic arch. After reflection of the skin flap, the temporal muscle was dissected from the temporal squama, and the anterior and posterior portions of the zygomatic arch were exposed and cut. The temporal muscle was reflected as far below the zygomatic arch as possible, and the whole temporal squama was exposed. Temporal craniotomy was performed and the extradural space of the temporal base was fully exposed. The well-encapsulated intracranial cystic mass was entirely located in the extradural space and was totally removed. The condyle was macroscopically intact, however, the anterior medial part of the mandibular fossa was completely destroyed, from which the lesion continued to the middle fossa. Subsequently, the right TMJ was also totally removed with condylectomy. A large number of cartilaginous bodies of various sizes were fully contained in the cystic mass. The intraoperative histological diagnosis was chondrosarcoma. Therefore, the surrounding tissues and bone of the temporal skull base were extensively removed. 
Postoperative histological examination showed various sizes of glistening blue/white ovoid loose bodies consisting of variable cellular hyaline cartilage covered with fine fibrous layers and synovial lining cells (Fig. 3A). The chondrocytes were clustered and had plump nuclei with some atypia such as pleomorphism or binucleate cells (Fig. 3B). Mitoses were rarely seen. The final histological diagnosis was chondromatosis of the TMJ.

Postoperative MR imaging confirmed total removal of the tumor. No additional adjuvant therapy was performed. Intermittent fixation of the TMJ and elastic training were performed. The patient was discharged 2 weeks after surgery. He had no dysfunction of the right TMJ and has so far shown no signs of tumor recurrence.

\section{Discussion}

Synovial chondromatosis arising from the TMJ with extension into the intracranial space is extremely rare, with only 8 reported cases. ${ }^{2,5,6,8-12)}$ The clinical features of the disease are still unclear. Table 1 summarizes the clinical features, diagnoses, and surgical strategies of the 9 cases (including our case). The patients with synovial chondromatosis with intracranial extension were aged 41 to 64 years (mean 52.6 years), whereas patients with disease restricted to the TMJ were 21 to 66 years (mean 55.4 years). The affected side was the right in 4 patients and the left in 5 (disease only in the TMJ, right/left 31/37). There were 5 males and 4 females with synovial chondromatosis in the TMJ with intracranial extension, compared to 49 males and 24 females with disease restricted in the TMJ. Therefore, the clinical features matched except for the sex distribution.

The predominant symptoms of synovial chondromatosis in the TMJ with intracranial extension were pain and swelling (8/9), restriction of joint movement (2/9), and clicking sound on opening the mouth (2/9). In addition, 3 patients showed 7 th and/or 5th cranial nerve disturbance, and 3 patients had hearing loss caused by involvement of the external auditory canal or Eustachian tube. Such cranial nerve involvement indicates that the disease has reached the advanced stage.

Preoperative diagnosis was difficult to establish despite CT and MR imaging in all cases (Table 1). The preoperative diagnosis was correct in only 2 cases. The other diagnoses were joint derangement or chondrosarcoma, even using the preoperative needle biopsy technique.

CT delineates a well-circumscribed water-density mass with multiple finely stippled calcification enclosed by the temporomandibular capsule. ${ }^{16)} \mathrm{CT}$ can also reveal associated erosion of the glenoid fossa and intracranial involvement. ${ }^{8)}$ The mass appears as mixed density containing both fluid and solid materials. The solid portion might be attributed to neoplasia of the mesenchymal tissue remnants originating from the synovial membrane, and the fluid part to synovial secretion accumulated with the joint space. ${ }^{16)}$ MR imaging can clearly show the extension of the mass into the middle cranial fossa and homogeneous intensity area isointense with the temporal lobe on both $\mathrm{T}_{1^{-}}$and $\mathrm{T}_{2}$-weighted images. ${ }^{15)} \mathrm{In}$ our case, a clear margin of the mass was identified between the mass and the temporal lobe, which indicated no dural disruption and cortical invasion.

The surgical strategy in our patient used the combined trans-zygomatic temporal skull base and preauricular approaches. Various surgical approaches have been adopted such as the pre-auricular approach combined with middle fossa craniotomy, trans-zygomatic craniotomy, and others. ${ }^{2,5,6,8-12)}$ One patient underwent two-staged surgery. As the intracranial mass arising from the TMJ is located in the temporal base, extensive exposure of the temporal skull base is beneficial for total removal of the intracranial mass. For this purpose, the trans-zygomatic temporal skull base approach by neurosurgeons is highly advantageous. In particular, a complete extradural approach is very successful, and opening the dura and direct retraction of the temporal lobe are unnecessary. After removal of the extradural mass in the temporal skull base, the lesion in the TMJ can be removed via a pre-auricular approach. We believe that this combined trans-zygomatic temporal skull base and pre-auricular approach is the best approach for synovial chondromatosis in the TMJ with intracranial extension. The mandibular function is generally not affected by condylectomy, however, the possibility of disorder of dental articulation still remains when the patients lose their teeth with aging. No local recurrence or distal metastasis has been reported after total removal of the lesion. No adjuvant therapy such as chemotherapy and/or radiation therapy is needed. Local recurrences have been reported after incomplete removal of the mass and synovectomy. ${ }^{1,13)}$ Therefore, total removal of the lesion is recommended at the first operation.

Establishing the histological diagnosis of synovial chondromatosis in the TMJ is difficult, and misdiagnosis as chondrosarcoma is easy. Synovial chondromatosis in the TMJ is quite rare, and chondrocytes in synovial chondromatosis sometimes show nuclear atypia such as pleomorphism and binuclear cells, which indicates malignancy.7) Pathologists should be aware of such histological characteristics 


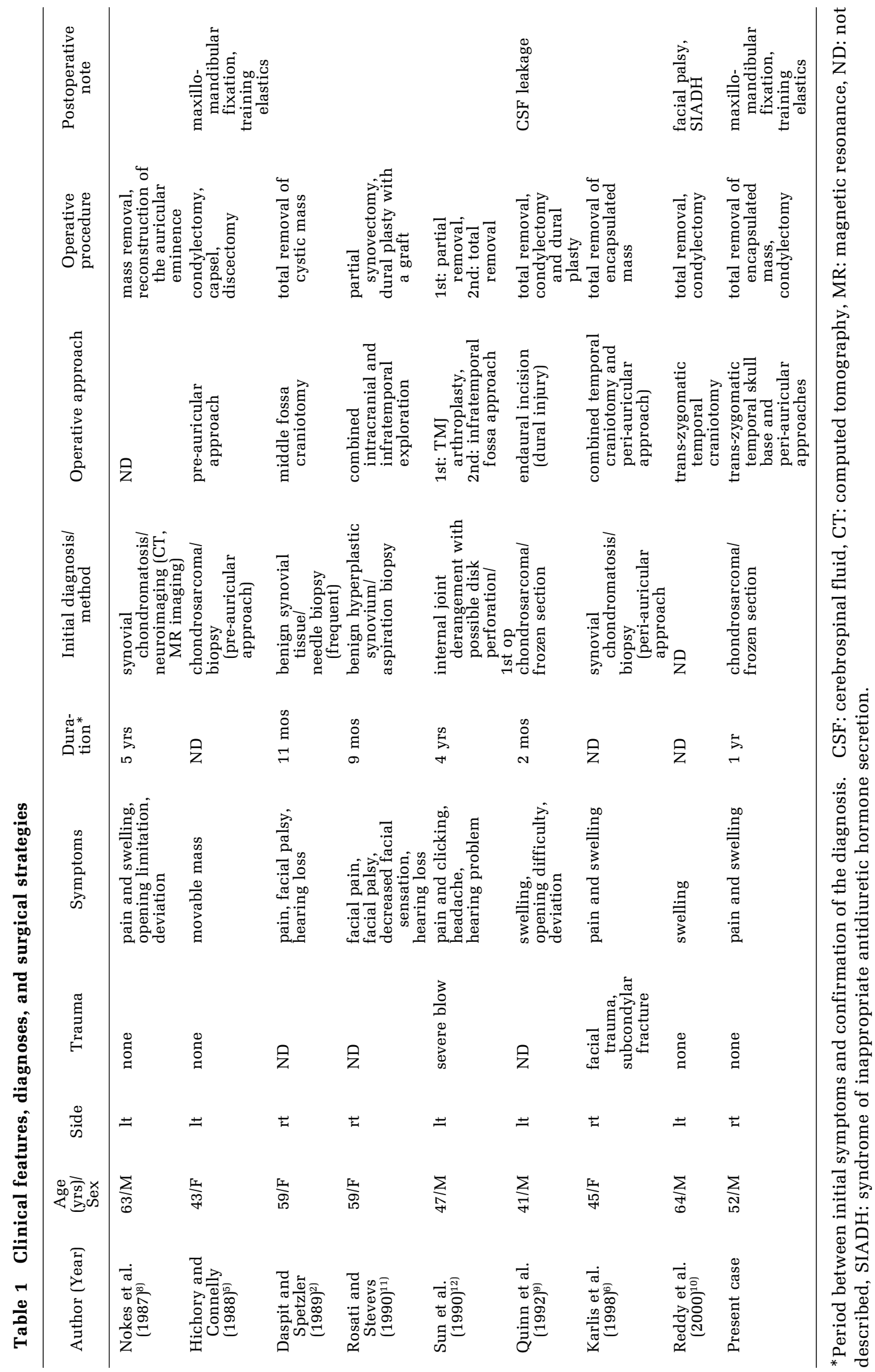


and avoid over-diagnosis. A large number of ovoid loose bodies of various sizes are packed closely in the cystic mass appearing like grains of rice in sushi. The key point in the correct diagnosis of synovial chondromatosis is to intraoperatively identify ovoid loose bodies in the TMJ. Preoperative CT and MR imaging performed by well-trained neuroradiologists, with experience and/or awareness of the disease, could discriminate chondrosarcoma and synovial chondromatosis. However, total removal of the lesion including the TMJ and finding the loose bodies are necessary to confirm the diagnosis and achieve cure of synovial chondromatosis.

The present case of synovial chondromatosis in the TMJ with extension into the middle cranial fossa was surgically treated via combined trans-zygomatic temporal skull base and pre-auricular approaches. The lesion is extremely rare and is histologically very difficult to discriminate from chondrosarcoma. Preoperative and/or intraoperative diagnosis using biopsy or surgical specimen is indispensable for optimum treatment.

\section{Acknowledgments}

We express sincere appreciation to Dr. Hideo Tanaka, Division of Oral Surgery and Pathology, Hamamatsu University School of Medicine, for helpful advise and technical assistance.

\section{References}

1) Blankestijn J, Panders AK, Vermey A, Scherpbier AJ: Synovial chondromatosis of the temporo-mandibular joint. Report of three cases and a review of the literature. Cancer 55: 479-485, 1985

2) Daspit CP, Spetzler RF: Synovial chondromatosis of the temporomandibular joint with intracranial extension. Case report. J Neurosurg 70: 121-123, 1989

3) Ginaldi S: Computed tomography feature of synovial osteochondromatosis. Skeletal Radiol 5: 219-222, 1980

4) Haller A: Elementa Physiologiae Corporus Humani. Bernae, 1776

5) Hickory JE, Connelly MV: Two-stage reconstruction of the temporomandibular joint following treatment of synovial chondromatosis. J Oral Maxillofac Surg 46: 617-619, 1988
6) Karlis V, Glickman RS, Zaslow M: Synovial chondromatosis of the temporomandibular joint with intracranial extension. Oral Surg Oral Med Oral Pathol Oral Radiol Endod 86: 664-666, 1998

7) Miller MV KA, Mertens F: World Health Organization Classification of Tumors. Pathology and Genetics of Tumors, Soft Tissue and Bone. Lyon, IARC Press, 2002

8) Nokes SR, King PS, Garcia R Jr, Silbiger ML, Jones JD, Castellano ND: Temporomandibular joint chondromatosis with intracranial extension: MR and CT contributions. AJR Am J Roentgenol 148: 1173-1174, 1987

9) Quinn PD, Stanton DC, Foote JW: Synovial chondromatosis with cranial extension. Oral Surg Oral Med Oral Pathol 73: 398-402, 1992

10) Reddy PK, Vannemreddy PS, Gonzalez E, Nanda A: Synovial chondromatosis of the temporomandibular joint with intracranial extension. J Clin Neurosci 7: 332-334, 2000

11) Rosati LA, Stevens C: Synovial chondromatosis of the temporomandibular joint presenting as an intracranial mass. Arch Otolaryngol Head Neck Surg 116: 1334-1337, 1990

12) Sun S, Helmy E, Bays R: Synovial chondromatosis with intracranial extension. A case report. Oral Surg Oral Med Oral Pathol 70: 5-9, 1990

13) Takagi M, Ishikawa G: Simultaneous villonodular synovitis and synovial chondromatosis of the temporomandibular joint: report of case. J Oral Surg 39: 699-701, 1981

14) von Lindern JJ, Theuerkauf I, Niederhagen B, Berge S, Appel T, Reich RH: Synovial chondromatosis of the temporomandibular joint: clinical, diagnostic, and histomorphologic findings. Oral Surg Oral Med Oral Pathol Oral Radiol Endod 94: 31-38, 2002

15) Wong WC, Cheng PW, Chan FL: MRI appearance of synovial chondromatosis in the temporomandibular joint. Clin Radiol 56: 773-774, 2001

16) Yu Q, Yang J, Wang P, Shi H, Luo J: CT features of synovial chondromatosis in the temporomandibular joint. Oral Surg Oral Med Oral Pathol Oral Radiol Endod 97: 524-528, 2004

Address reprint requests to: Naoki Yokota, M.D., Department of Neurosurgery, Hamamatsu University School of Medicine, 1-20-1 Handayama, Higashiku, Hamamatsu, Shizuoka 431-3192, Japan. e-mail: yokota@hama-med.ac.jp 